\title{
COMMENT Resistance of children to Covid-19. How?
}

\author{
Alain Fischer ${ }^{1,2,3}$ \\ Mucosal Immunology (2020) 13:563-565; https://doi.org/10.1038/s41385-020-0303-9
}

The Covid-19 pandemic has emerged in China in late $2019{ }^{1}$ Within 5 months, more than 4,7 million people have been infected. Two to five percent of them developed a severe acute respiratory syndrome (SARS) that can be associated with multiorgan failure and causes death overall in $\sim 0.5 \%$ of infected patients that is over 315,000 people at time of this writing. ${ }^{1-4}$ It was rapidly found that Covid- 19 is caused by a $\beta$ coronavirus denominated SARS-Cov-2 that is related to SARS-Cov-1. ${ }^{5}$ Not all individuals are equal in terms of risk of infections and lethality. The very first reports of Chinese cohorts showed that age was a strong risk factor since median age at death was over 80 years, while the fatal risk dramatically increases as a function of age. ${ }^{1,2}$ It was found that children did not account for more that $1-2 \%$ of infected cases and that casualties were minimal, although not absent. ${ }^{6-11}$ Data from several regions of the world (China, Italy, U.S., and France) have reported similar figures. ${ }^{1-4}$ There are actually two intriguing features:

(i) It looks like the risk of getting the virus, as estimated in screening studies in individuals at risk because they were in contact with infected patients is lower in children than in adults, that is 1.3 vs. $3.5 \%$ in one study. ${ }^{10}$ These figures have to be taken cautiously because of possible bias, but they suggest that children may to some extent be resistant to the infection.

(ii) Once infected, the risk for a child to develop severe disease requiring hospitalization is 25 times lower than in adults $(0.1 \%$ vs. $2.6 \%)$ and the risk of death is 500 times lower than in adults $(0.001 \%$ vs. $0.5 \%)$ as based on a recently published French study. ${ }^{4}$

Thus, both resistance to infection and resistance to disease appear to be much stronger in children than in adults. The apparent resistance to infection might actually reflect a more rapid clearance of the virus so that the chance to detect cases is diminished. Future studies on seropositivity prevalence should help to distinguish between these possibilities. Of note the increased male to female ratio as observed in adult Covid-19 patients (6 to 4) is also observed in children. Finally, children below the age of 1 year are over-represented in pediatric Covid-19 cohorts with a higher risk of fatality compared with children above the age of 1 .

It is thus appropriate to try to understand why, overall children are less prone to Covid-19. The first remark is that this resistance is not specific to that particular virus. For instance and it is likely relevant, no children died from infection by SARS-Cov-1, a related $\beta$ coronavirus. $^{12}$ Considering other viral infections, it is well known that primary varicella zoster virus infection is much more symptomatic in adults than in children with persistent fever and fatigue. Also, Epstein-Barr virus infection is in most cases entirely asymptomatic in children notably below the age of 4 years while it causes infectious mononucleosis in teenagers and adults with long lasting general symptoms such a fever and fatigue. ${ }^{13}$ Influenza infection affects children as well as adults, unlike SarsCov2 as mentioned above but it is much less frequently severe or life-threatening than in elderly people, although lethality in children is higher in proportion as compared with Covid-19. ${ }^{14}$

Several factors need to be taken into consideration but, as indicated none of them provide yet formal clues to understand this resistance of children to Covid-19. Let's examine the hypotheses.

\section{"RESISTANCE" TO INFECTION?}

Some have argued that it does not correspond to a true resistance but rather to a reduced exposure to infected people who are excreting high viral particles because children experience less outdoor activities and travel less as compared with adults. ${ }^{11}$ Exposure is however not a major parameter as the infection rate estimated within a given school in an early infection hot spot in France reached $38 \%$ of pupils (15-17 years old) as based on antibody detection.

It has been proposed that the biology of the viral receptor, i.e., angiotensin converting enzyme $2(\mathrm{ACE})^{15}$ differs in children. A report mentions that its expression may be higher in children (that is rather counterintuitive to resistance) but no clear data are shown. ${ }^{11}$ Overall, this could nevertheless fit with the fact that acute pneumonia caused by coronavirus H6VNL63 that also binds to ACE2 is more common in adults than in very young children. ${ }^{16}$

\section{A MORE RAPID CONTROL OF INFECTION?}

Immunity to virus obviously combines an early innate immune response followed by adaptive $T$ and $B$ cell responses. Today, knowledge on which effector mechanisms are involved in SARSCov-2 infection is lacking. It is very likely that SARS-Cov-2-a single stranded RNA (ssRNA) virus-innate immune response through ssRNA binding in the cytosol to sensors such a RIG I and, in endosomes, to Toll like receptor (TLR) 3, rapidly inducing a potent antiviral response through induction of interferons type I and possibly type III on mucosal surfaces. ${ }^{17-21}$ It would thus be of great interest to compare (a) in vitro characteristics of interferon type I production by children/adult cells such as plasmacytoid dendritic cells (pDC) ${ }^{22}$ but also monocytes upon challenge with SARS-Cov-2 and to assess interferon detection and responses (expression of interferon signaling genes) in infected children

\footnotetext{
${ }^{1}$ Collège de France, Paris, France; ${ }^{2}$ Immunology and Pediatric Hematology Department, Assistance Publique-Hôpitaux de Paris, Paris, France and ${ }^{3}$ INSERM UMR 1163 , Institut Imagine, Paris, France

Correspondence: Alain Fischer (alain.fischer@aphp.fr)
}

Received: 5 May 2020 Accepted: 8 May 2020

Published online: 28 May 2020 
compared with adults. Likewise, given the potential antiviral role of interferon type III, comparison of epithelial cells derived from adult and pediatric organoid material could be of interest. ${ }^{23}$ At the opposite of the age spectrum, it has been reported that aging impairs interferon response induced by TLR9. ${ }^{24}$ Interestingly enough, it has recently been shown that respiratory syncytial virus (RSV) infection is significantly milder-that is not requiring hospitalization-in young children who exhibited a high type I interferon response. ${ }^{25}$ Although correlative, these data pinpoint to a critical role of interferon in an early stage of RSV control. This may be relevant to Sars-Cov2 as the latter virus has been shown to potently inhibit in vitro and in an animal ferret model type I and III interferon responses, suggesting that an interferon response is a critical factor for infection control as its intensity could determine the outcome by overriding the virus-mediated inhibitory activity. ${ }^{26}$

If we assume that, indeed, appropriate immune responses to SARS-Cov-2 are more rapid and efficient in children, this could still be related either to an intrinsic (developmentally programmed) higher capacity or an acquired predisposition. It is tempting to speculate that the recurrence of viral infections of the upper respiratory tract in children could trigger some forms of trained immunity whereby monocytes and other myeloid cells underwent (at the progenitor cell state) some epigenetic imprinting that make them prone to produce effective immune mediators. ${ }^{27}$ Such trained immunity phenomenon has been found at work in experimental murine models following BCG on candida infections. Data in humans are more scarce but there is some suggestions that trained immunity may exist. This again should be testable.

There is an adaptive immune response to SARS-Cov-2 since antibodies can be detected from 6 to 7 days after infection. ${ }^{4}$ Their protective role as well as the one of $T$ cell responses, including the polarization status of effector T cells will need to be studied in detail. Whether such responses may differ in terms of kinetics, pattern, and intensity between children and adults is a matter of speculation. As it is the case for other viruses, the capacity of elderly people who are at higher risk of developing a severe Covid-19 disease to mount an effective adaptive immune response is reduced, a mirror risk factor at the opposite spectrum of age.

\section{A LESS INTENSE IMMUNOPATHOLOGICAL REACTION}

Severe Covid-19 disease is associated with a cytokine release syndrome (CRS) with high concentrations of notably IL6, TNF, and chemokines detected in blood and tissues. ${ }^{28-30}$ One report showed that Covid-19 infected children displayed only a moderate increase of IL6 (and IL17F). ${ }^{10}$ Cellular sources likely include at least phagocytic cells that massively migrate to the lung ${ }^{28-30}$ but may include many other types of cells that produce IL6, such as epithelial, endothelial, and lymphoid cells. What is unknown is whether the CRS is a primary response of myeloid cells to the viral infection or is a secondary $T$ cell-triggered response as observed in CRS complicating treatment of cancer with chimeric antigen receptor T cells. ${ }^{28}$ Of interest, is the fact that most comorbidity known in adults to increase the risk of ARDS and multiorgan failure caused by SARS-Cov-2 infection, i.e., cardiovascular disease, obesity, and type II diabetes, are known be associated with protracted low-grade inflammation. ${ }^{31-33}$ It may thus be that myeloid cells (or other types of cells), poised to inflammation because of metabolic alteration and/or microbiota dysbiosis $^{31}$ are overreacting in the setting of the infection. Children rarely present such comorbidities, thus could be less exposed to the risk of hyper inflammation. According to that hypothesis, children would be less susceptible to severe Covid19 exactly as healthy adults are, not because of specific characteristics of childhood!

\section{A BETTER CAPACITY OF TISSUE REPAIR}

Resistance to disease implies on one hand the fight against the triggering factor and on the other hand the capacity of dampening disease consequences, i.e., direct cell toxicity and/or immunopathology. ${ }^{34}$ Many factors are involved in tissue preservation/repair such as efferocytosis (noninflammatory cell death) and related switch of monocytes/macrophages to a so called M2 repair program. ${ }^{35,36}$ Lipids such as resolvins are known to play an important role in this process. There again, it may be proposed that, during childhood, there is a stronger intrinsic capacity of such pathways ("M2/M1 balance") and/or that, in adults they are impaired by comorbidity. It is for instance known that expression of resolvins is reduced in the context arteriosclerosis. $^{37}$

It could well be that all four above discussed mechanisms concur to protect children from SARS-Cov-2 infection. But we should admit that our present knowledge is far too limited to propose any model. At least, obvious investigations in infected individuals as well as in vitro experiments and possibly animal models will provide some answers.

\section{WHAT ABOUT CHILDREN AT RISK FOR SEVERE COVID-19}

Children below the age of 1 year account for a higher than expected number of cases of Covid-19 including a few severe cases.8 Obviously, this observation would suggest a role of immaturity in immune responses-as observed for Herpex simplex infection ${ }^{38}$-or an absence yet of trained immunity as discussed above!

A number of inherited diseases ( $>420$ ) affect the human immune system. ${ }^{39}$ Some are associated with severe predisposition to infectious agents that are either broad or in contrast limited such as IRF7 and IRF9 deficiencies that are associated with severe influenza infection. ${ }^{40,41}$ These cases are extremely rare but it might be important-both for these patients-and for deciphering key effector mechanisms of immunity to SARS-Cov-2, to identify which genetic defects are predisposing to severe Covid-19. At the time of this writing, no such report can be found in the literature. Obviously, to search by whole-exome (or genome) sequencing for a genetic anomaly in the extremely rare severe cases observed in children, adolescents and even young adults might provide interesting clues. Conversely, given the pathogenic role of hyper inflammation, it would be important to see whether patients with autoinflammatory syndromes associated with high IL-1 production are at higher risk. ${ }^{42}$ The same is true in a setting for hypereactivity to IL6 caused by gain-of-function mutations of STAT3. ${ }^{43}$ This has not been reported yet. It would also be of interest to know whether patients with "interferonopathies" a group of diseases that share in common constant and high production of type I interferon are a protective (or a risk) factor for severity of Covid-19.44 Collection of rare observations in these settings would be extremely valuable.

Very recently, in addition, an alert has been passed about the outbreak of Kawasaki like syndrome cases in children in the context of Covid-19. ${ }^{45}$ It is too early to assess the risk factor and the pathophysiology, although an immunopathological reaction to a virus able to infect endothelial cells could be considered.

In conclusion, much has to be learned to understand the major differences seen in the handling of SARS-Cov-2 between children and adults, especially elderly people. Are there specific protection factors in childhood or conversely specific risk factors in elderly or both? This is not only a theorical question as understanding susceptibility/resistance mechanisms can contribute to design therapy and more importantly vaccine.

\section{AUTHOR CONTRIBUTIONS}

Alain Fischer wrote the review based on his assessment of relevant bibliography and his thoughts. 


\section{ADDITIONAL INFORMATION}

Competing interests: The author declares no competing interests.

Publisher's note Springer Nature remains neutral with regard to jurisdictional claims in published maps and institutional affiliations.

\section{REFERENCES}

1. Zhu, N. et al. A novel coronavirus from patients with pneumonia in China, 2019. N. Engl. J. Med. 382, 727-733 (2020).

2. Guan, W. J. et al. Clinical characteristics of coronavirus disease 2019 in China. $N$. Engl. J. Med. 382, 1708-1720 (2020).

3. Report on Covid-19 MRW. 69, 344-346 (2020).

4. Salje, H. et al. Estimating the burden of SARS-CoV-2 in France. Science. https://doi. org/10.1126/science.abc3517 (2020).

5. Zhou, P. et al. A pneumonia outbreak associated with a new coronavirus of probable bat origin. Nature 579, 270-273 (2020).

6. Wu, Z., McGoogan, J. M. Characteristics of and important lessons from the coronavirus disease 2019 (COVID-19) outbreak in China: summary of a report of 72314 cases from the Chinese Center for Disease Control and Prevention. JAMA. 323, 1239-42 (2020).

7. Lu, X. et al. SARS-CoV-2 Infection in Children. N. Engl. J. Med. 382, 1663-1665 (2020).

8. Dong, Y. et al. Epidemiology of COVID-19 among children in China. Pediatrics. https://doi.org/10.1542/peds.2020-0702 (2020).

9. Cai, J. et al. A Case Series of children with 2019 novel coronavirus infection: clinical and epidemiological features. Clin. Infect. Dis. https://doi.org/10.1093/cid/ ciaa198 (2020)

10. $\mathrm{Xu}, \mathrm{Y}$. et al. Characteristics of pediatric SARS-CoV-2 infection and potential evidence for persistent fecal viral shedding. Nat. Med. 26, 502-505 (2020).

11. Lee, P. I., Hu, Y. L., Chen, P. Y., Huang, Y. C., Hsueh, P. R. Are children less susceptible to COVID-19? J. Microbiol. Immunol. Infect. https://doi.org/10.1016/j. jmii.2020.02.011 (2020).

12. Kliegman, R., St Geme, J., Blum, N. H. Nelson Textbook of Pediatrics 20th edn (Elsevier Limited, 2020)

13. Taylor, G. S., Long, H. M., Brooks, J. M., Rickinson, A. B. \& Hislop, A. D. The immunology of Epstein-Barr virus-induced disease. Annu Rev. Immunol. 33, 787-821 (2015)

14. Principi, N. \& Esposito, S. Severe influenza in children: incidence and risk factors. Expert Rev. Anti Infect. Ther. 14, 961-968 (2016).

15. Lu, R. et al. Genomic characterisation and epidemiology of 2019 novel coronavirus: implications for virus origins and receptor binding. Lancet 395, 565-74. (2020).

16. Huang, S. H. et al. Epidemiology of human coronavirus NL63 infection among hospitalized patients with pneumonia in Taiwan. J. Microbiol. Immunol. Infect. $\mathbf{5 0}$ 763-70. (2017).

17. Muller, U. et al. Functional role of type I and type II interferons in antiviral defense. Science 264, 1918-1921 (1994).

18. Janeway, C. A. Jr. \& Medzhitov, R. Innate immune recognition. Annu. Rev. Immunol. 20, 197-216 (2002).

19. Rehwinkel, J., Gack, M. U. RIG-I-like receptors: their regulation and roles in RNA sensing. Nat. Rev. Immunol. https://doi.org/10.1038/s41577-020-0288-3 (2020).

20. Sioud, M. Innate sensing of self and non-self RNAs by Toll-like receptors. Trends Mol. Med. 12, 167-176 (2006).

21. Ziegler, C. G. K. et al. SARS-CoV-2 receptor ACE2 is an interferon-stimulated gene in human airway epithelial cells and is detected in specific cell subsets across tissues. Cell. https://doi.org/10.1016/j.cell2020104.0315 (2020).
22. Reizis, B. Plasmacytoid dendritic cells: development, regulation, and function Immunity 50, 37-50 (2019).

23. Lamers, M. M. et al. SARS-CoV-2 productively infects human gut enterocytes. Science. https://doi.org/10.1126/science.abc1669 (2020).

24. Stout-Delgado, H. W., Yang, X., Walker, W. E., Tesar, B. M. \& Goldstein, D. R. Aging impairs IFN regulatory factor 7 up-regulation in plasmacytoid dendritic cells during TLR9 activation. J. Immunol. 181, 6747-6756 (2008).

25 . Heinonen, S. et al. Immune profiles provide insights into respiratory syncytial virus disease severity in young children. Sci. Transl. Med. 12, https://doi.org/ 10.1126/scitransImed.aaw0268 (2020).

26. Blanco-Melo D., et al. Imbalanced hostresponse to SARS-CoV-2 drives development of COVID-19. Cell. https://doi.org/10.1016/j-cell202004-026 (2020).

27. Netea, M. G. et al. Defining trained immunity and its role in health and disease. Nat. Rev. Immunol. https://doi.org/10.1038/s41577-020-0285-6 (2020).

28. Mehta, P. et al. COVID-19: consider cytokine storm syndromes and immunosuppression. Lancet 395, 1033-1034 (2020).

29. Yang, X. et al. Clinical course and outcomes of critically ill patients with SARS-CoV2 pneumonia in Wuhan, China: a single-centered, retrospective, observational study. Lancet Respir. Med. 8, 475-81 (2020).

30. Pedersen, S. F., Ho, Y. C. SARS-CoV-2: a storm is raging. J Clin. Investig. 130, 2202-5 (2020).

31. Galkina, E. \& Ley, K. Immune and inflammatory mechanisms of atherosclerosis (*). Annu. Rev. Immunol. 27, 165-97 (2009).

32. Saltiel, A. R. \& Olefsky, J. M. Inflammatory mechanisms linking obesity and metabolic disease. J. Clin. Investig. 127, 1-4 (2017).

33. Cox, A. J., West, N. P. \& Cripps, A. W. Obesity, inflammation, and the gut microbiota. Lancet Diabetes Endocrinol. 3, 207-215 (2015).

34. Soares, M. P., Teixeira, L. \& Moita, L. F. Disease tolerance and immunity in host protection against infection. Nat. Rev. Immunol. 17, 83-96 (2017).

35. Henson, P. M. Cell removal: efferocytosis. Annu Rev. Cell Dev. Biol. 33, 127-44. (2017).

36. Linton, M. F. et al. Macrophage apoptosis and efferocytosis in the pathogenesis of atherosclerosis. Circ. J. 80, 2259-68. (2016).

37. Serhan, C. N. \& Levy, B. D. Resolvins in inflammation: emergence of the pro resolving superfamily of mediators. J. Clin. Investig. 128, 2657-69. (2018).

38. Giraldo, D., Wilcox, D. R. \& Longnecker, R. The type I interferon response and agedependent susceptibility to herpes simplex virus infection. DNA Cell Biol. 36, 329-34. (2017).

39. Tangye, S. G. et al. Human inborn errors of immunity: 2019 update on the classification from the international union of immunological societies expert committee. J. Clin. Immunol. 40, 24-64 (2020).

40. Ciancanelli, M. J. et al. Infectious disease. Life-threatening influenza and impaired interferon amplification in human IRF7 deficiency. Science 348, 448-453 (2015).

41. Hernandez, N. et al. Life-threatening influenza pneumonitis in a child with inherited IRF9 deficiency. J. Exp. Med. 215, 2567-85. (2018).

42. de Jesus, A. A., Canna, S. W., Liu, Y. \& Goldbach-Mansky, R. Molecular mechanisms in genetically defined autoinflammatory diseases: disorders of amplified danger signaling. Annu. Rev. Immunol. 33, 823-874 (2015).

43. Fabre, A. et al. Clinical aspects of STAT3 gain-of-function germline mutations: a systematic review. J. Allergy Clin. Immunol. Pr. 7, 1958-69 e9 (2019).

44. Uggenti, C., Lepelley, A. \& Crow, Y. J. Self-awareness: nucleic acid-driven inflammation and the type I interferonopathies. Annu. Rev. Immunol. 37, 247-67. (2019).

45. Viner, R. M., Whittaker, E. Kawasaki-like disease: emerging complication during the COVID-19 pandemic. Lancet. (2020). 